\title{
Ligações e transmissão de volatilidade intradiária entre mercados bolsistas europeus no âmbito da crise financeira global
}

Vitor Manuel de Sousa Gabriel*

José Ramos Pires Manso ${ }^{* *}$

\section{Abstract}

This study analyzes the short-term connections and the transmission of intraday volatility across seven European markets, particularly Germany (DAX), Spain (IBEX 35), France (CAC 4O), Greece (ATG), Ireland (ISEQ), Portugal (PSI 20) and the UK (FTSE 100), from 24/01/200o to 30/06/2011. This study uses a vector autoregressive model, the concept of Granger causality and impulse response functions, in order to understand whether the global financial crisis changes the shortterm connections and the intraday volatility transmission process.

\section{Keywords}

Global financial crisis, European stock markets, intraday volatility transmission, vector autoregressive.

\section{JEL Classification}

C32, C58, G15.

\begin{abstract}
Resumo prazo e os mecanismos de transmissão de volatilidade intradiária entre sete mercados europeus, concretamente dos mercados da Alemanha (DAX), da Espanha (IBEX 35), da França (CAC 40), da Grécia (ATG), da Irlanda (ISEQ), de Portugal (PSI 20) e do Reino Unido (FTSE 100), no período compreendido entre 24/1/2000 e 30/6/2011. A análise recorre a um vetor autorregressivo, ao conceito de causalidade de Granger e a funções de impulso-resposta, com o objetivo de perceber se a recente crise financeira global provocou alterações ao nível das ligações de curto prazo e dos mecanismos de transmissão de volatilidade intradiária.
\end{abstract}




\section{1_Introdução}

Ao longo das últimas décadas, o mundo assistiu a diversas crises financeiras, com origem em determinadas regiões e países, que se estenderam geograficamente. De acordo com diversos autores, entre os quais Claessens et al. (2010), Bekaert et al. (2011) e Lin e Treichel (2012), a atual crise financeira é a primeira verdadeiramente global e a mais severa crise desde a Grande Depressão. Embora tivesse origem nos EUA, designadamente com a crise do crédito subprime, acabaria por se transmitir a outros setores econômicos, bem como a outras economias, quer desenvolvidas, quer emergentes. Também viria a afetar as bolsas mundiais, com muitos desses mercados a viverem crashes mais acentuados do que o dos próprios EUA.

A interligação entre diferentes mercados tem sido, desde há muito tempo, um tópico de estudo de acadêmicos. À medida que os mercados internacionais se tornam mais integrados, a informação gerada num mercado pode afetar outros mercados. Diversos estudos têm sido desenvolvidos acerca do comovimento de longo prazo, das ligações dinâmicas das rendibilidades e das transmissões de volatilidade entre os mercados financeiros internacionais.

Os estudos pioneiros acerca da transmissão internacional de choques nas rendibilidades inspiraram-se no trabalho de Eun e Shim (1989), que analisou os mecanismos de transmissão internacional de movimentos entre nove mercados bolsistas, através de um sistema autorregressivo, para concluir pela existência de fortes interdependências entre os mercados, com o mercado dos EUA a revelar-se o mais influente. Yang et al. (2003) recorreram a uma análise de correlação contemporânea e a um vetor autorregressivo, com base no qual foram estimadas funções de impulso-resposta, para concluírem que as ligações entre os mercados europeus se tornaram mais fortes com a União Econômica e Monetária. A ocorrência de efeitos do tipo lead-lag entre mercados, como os identificados nesses estudos, pode ser encarada como uma quebra da hipótese do mercado eficiente, de acordo com a qual os preços dos ativos não são previsíveis, comportando-se como um passeio aleatório (Fama, 1970).

No início da década de 1990, alguns acadêmicos começaram a dar importância à modelização de interações entre mercados bolsistas. Hamao et al. (1990) estudaram os mercados dos EUA, do Reino Unido e do Japão, entre 1985 e 1988, recorrendo a um modelo GARCH, para concluir pela ocorrência de contágio de volatilidade dos EUA para o Japão e do Reino Unido para o Japão, enquanto do Japão para os outros dois mercados o efeito de contágio se revelou débil. Booth et al. (1997) recorreram a um modelo de heterocedasticidade multivariado para estudar a transmissão de volatilidade entre os mercados bolsistas da Dinamarca, da Noruega, da Suécia e da Finlândia, entre 1988 e 1994, tendo sido identificadas algumas situações de efeito de contágio, com significância estatística, embora em reduzido número. Caporale et al. (2006), ao analisarem a transmissão de volatilidade entre alguns mercados europeus, do Japão e dos EUA, no período compreendido entre 1986 e 2000, recorrendo a um modelo GARCH-BEKK, identificaram a existência de efeitos de contágio de volatilidade. A ligação de causalidade entre os mercados, porém, foi de tipo unidirecional e teve origem nos mercados que viveram crises financeiras.

Diferentes metodologias têm sido consideradas na análise da transmissão de volatilidade. A primeira categoria envolve o recurso a modelos autorregressivos de heterocedasticidade condicional, inspirados no trabalho pioneiro de Bollerslev (1986). A segunda categoria é a dos chamados "modelos de mudança de regime”. A última categoria é a dos modelos estocásticos, introduzidos por Taylor (1982). 
Dos múltiplos estudos desenvolvidos acerca da temática da volatilidade, a maioria considera informação diária. Segundo alguns autores, porém, como Andersen e Bollerslev (1998), Alizadeh; Brandt; Diebold (1999), Beltratti e Morana (1999), Gallant; Hsu e Tauchen (1999) e Poon e Granger (2003), a consideração do valor de fecho dos ativos ou dos mercados ignora informação relevante sobre a trajetória dos preços.

Neste trabalho, recorremos à estimativa de Rogers; Satchell e Yoon (1994), que pressupõe a utilização de informação intradiária, nomeadamente os valores referentes à abertura, ao mínimo, ao máximo e ao fecho, para gerar as séries de volatilidade intradiária de sete índices europeus, mas também a um vetor autorregressivo (VAR), que serve de base à aplicação de testes de causalidade e de funções de impulso-resposta, com o objetivo de analisar as ligações de curto prazo e os mecanismos de transmissão de informação entre os mercados estudados.

O presente estudo segue a seguinte estrutura: a parte 2 apresenta a informação acerca dos dados e da metodologia escolhida; a parte 3 apresenta os resultados empíricos, ao passo que a parte 4 expõe, de forma resumida, as principais conclusões.

\section{2_Dados e metodologia}

\section{1_Dados}

No sentidodeanalisar aligação e a transmissão devolatilidade intradiária nos mercados europeus, foram selecionados índices representativos dos mercados da Alemanha (DAX 30), da França (CAC 40), do Reino Unido (FTSE 100), da Espanha (IBEX 35), da Irlanda (ISEQ Overall), da Grécia (ATG) e de Portugal (PSI 20).
Os dados utilizados neste estudo foram obtidos na Econostats (www.econostats.com) e cobrem o período compreendido entre 24 de janeiro de 2000 e 30 de junho de 2011, tendo sido subdividido em três subperíodos. Para analisar a crise Dot-Com, foi considerado o subperíodo de 24/1/2000 a 31/3/2003. Relativamente ao mais recente episódio de crise, que teve origem nos EUA, no setor do crédito subprime, $\mathrm{e}$ que viria a ter um novo epílogo com a crise das dívidas soberanas, considerou-se o dia $1^{\circ} / 8 / 2007$ como data de início, seguindo a indicação de Horta et al. (2008), Toussaint (2008) e Naoui et al. (2010), baseada na subida acentuada dos Credit Default Swaps. Para além dos subperíodos correspondentes a dois episódios de crise, foi ainda considerado um terceiro subperíodo, designado por Tranquilo, relativo ao lapso temporal de $1^{0} / 4 / 2003$ a 31/7/2007, e que correspondeu a uma subida generalizada dos valores dos índices das bolsas internacionais.

\section{2_Metodologia}

A metodologia selecionada no presente trabalho compreende as seguintes abordagens:

- Estimação de volatilidade intradiária através da proposta sugerida por Rogers; Satchell e Yoon (1994);

- Testes para a estacionariedade das séries;

- Correlações contemporâneas entre as volatilidades dos vários mercados;

- Significância estatística das correlações;

- Rácios de verossimilhança;

- Teste em duas amostras à igualdade de correlações;

- Estimação do VAR;

- Causalidade de Granger/Block exogeneity test;

- Funções de impulso-resposta 


\section{Estimativa de volatilidade intradiária}

Para estimar as séries de volatilidade intradiária, recorremos à metodologia de Rogers; Satchell e Yoon (1994), que incorpora um termo drift no processo estocástico e considera os preços de abertura $\left(O_{t}\right)$, de encerramento $\left(C_{t}\right)$, de máximo $\left(H_{t}\right)$ e de mínimo $\left(L_{t}\right)$, e que se traduz na seguinte expressão:

$$
\sigma_{r, t}^{2}=\sqrt{\frac{1}{N} \sum_{i=1}^{N}\left(\ln \frac{H_{t}}{C_{t}}\right)\left(\ln \frac{H_{t}}{O_{t}}\right)+\left(\ln \frac{L_{t}}{C_{t}}\right)\left(\ln \frac{L_{t}}{O_{t}}\right)}
$$

\section{Teste para a estacionariedade das séries}

O teste mais utilizado para apreciar a estacionariedade de uma série tem sido o sugerido por Dickey e Fuller (1979), que envolve o teste à integração das séries.

O teste de Dickey e Fuller Aumentado (ADF) pressupõe a consideração de expressões do tipo

$$
\begin{aligned}
& \Delta Y_{t}=\lambda^{*} Y_{t-1}+\delta_{1} \Delta Y_{t-1}+\delta_{2} \Delta Y_{t-2}+\ldots+\delta_{t-p+1}+u_{t} \\
& \Delta Y_{t}=\alpha+\lambda^{*} Y_{t-1}+\delta_{1} \Delta Y_{t-1}+\delta_{2} \Delta Y_{t-2}+\ldots+\delta_{t-p+1}+u_{t} \\
& \Delta Y_{t}=\alpha+\lambda^{*} Y_{t-1}+\delta_{1} \Delta Y_{t-1}+\delta_{2} \Delta Y_{t-2}+\ldots+\delta_{t-p+1}+u_{t}
\end{aligned}
$$

Em que $Y$ se refere à série estudada. As expressões anteriores são correções paramétricas, que consistem na adição de termos de diferenças defasadas, na equação de regressão, para corrigir a correlação em série de ordem superior.

No teste anterior, a formulação de hipóteses é expressa do seguinte modo:

$$
\begin{array}{cc}
H_{0}: \lambda=\beta=0 \quad \begin{array}{l}
\text { (a série } \mathrm{Y} \text { possui raiz unitária } \\
\text { ou a série é não estacionária) }
\end{array} \\
H_{a}: \lambda<0 \text { e } \beta<0 & \begin{array}{l}
\text { (a série } \mathrm{Y} \text { não possui raiz unitária } \\
\text { ou a série é estacionária) }
\end{array}
\end{array}
$$

\section{Análise de correlações}

A correlação da volatilidade intradiária de cada par de índices é proporcionada pela já conhecida expressão

$$
r=\frac{\sum_{i=1}^{n}\left(x_{i}-\bar{x}\right)\left(y_{i}-\bar{y}\right)}{\left[\sum_{i=1}^{n}\left(x_{i}-\bar{x}\right)^{2} \sum_{i=1}^{n}\left(y_{i}-\bar{y}\right)^{2}\right]}
$$

Para testar se o coeficiente de correlação é significativamente diferente de zero, recorre-se à estatística $t=\frac{r \cdot \sqrt{n-2}}{\sqrt{1-r^{2}}}$, que segue a distribuição t, com $n-2$ graus de liberdade, e em que $r$ é o coeficiente de correlação, e $n$ o número de observações.

Para testar se a matriz de coeficientes de correlação é globalmente diferente da matriz identidade, aplica-se o teste do rácio de verossimilhança (Pindick; Rotemberg, 1990). A hipótese nula desse teste pressupõe que, globalmente, não existe correlação entre os vários mercados da amostra. A estatística do teste é dada por $t=-N \cdot \log |R|$ e segue uma distribuição Qui-Quadrado, como, 0,5p(p-1) graus de liberdade, em que $|R|$ é o determinante da matriz de coeficientes de correlação, $N$ é o número de observações na amostra comum e $p$ é o número de séries analisadas no teste.

No sentido de se perceber se o aumento generalizado das correlações tem significância estatística, foi aplicado o teste em duas amostras, também designado de "teste $t$ de heterocedasticidade", e que resulta da proposta de Forbes e Rigobon (2002). Esse teste corresponde à hipótese nula de que a correlação no subperíodo Crise Financeira Global é superior ou igual à correlação nos dois subperíodos anteriores, contra a hipótese alternativa de que a correlação é superior durante os dois subperíodos precedentes.

$$
H_{0}=r_{i, j}^{1} \geq r_{i, j}^{0}
$$




$$
H_{1}=r_{i, j}^{1}<r_{i, j}^{0}
$$

Em que $r_{i, j}^{1}$ é o coeficiente de correlação entre o mercado i e o mercado j, no período t.

Na comparação entre o subperíodo Crise Financeira Global e os dois anteriores, estes últimos são designados por "0", e o subperíodo Crise Financeira Global é designado por "1".

A aplicação do teste recorre à transformação de Fisher dos coeficientes de correlação, de tal forma que esses apresentam uma distribuição aproximadamente normal, em termos assintóticos, com média, $\mu_{t}$, e variância, $\sigma_{t}^{2}$, definidas da seguinte forma:

$$
\begin{aligned}
& \mu_{t}=\frac{1}{2} \ln \left(\frac{1+r_{i, j}^{t}}{1-r_{i, j}^{t}}\right) \\
& \sigma_{t}^{2}=\frac{1}{n_{t}-3}
\end{aligned}
$$

A estatística do teste é calculada a partir de

$$
U=\frac{\bar{\mu}_{1}-\bar{\mu}_{0}}{\left(\sigma_{0}^{2}+\sigma_{1}^{2}\right)^{\frac{1}{2}}}
$$

Onde $\mu_{t}$ e $\sigma_{t}^{2}$ são a média e a variância amostrais, calculadas através da transformação de Fisher. A estatística do teste segue uma distribuição normal, com média 0 e variância 1 .

\section{Vetor autorregressivo}

Para se compreender o comportamento da volatilidade intradiária, é importante analisar as dinâmicas dos mercados, a transmissão e os mecanismos de propagação que guiam tais mercados. Para o efeito, é necessário um modelo que mostre claramente como a volatilidade é transmitida de um mercado a outro e que permita analisar a simultaneidade das interações entre os mercados. O Vetor Autorregres- sivo (VAR), desenvolvido por Sims (1980), é um dos modelos mais apropriados, permitindo estimar um sistema de equações dinâmicas simultâneas, sem estabelecer restrições prévias na estrutura das relações entre as variáveis.

O presente estudo expressa o modelo VAR do seguinte modo:

$$
Y_{t}=C+\sum_{s=1}^{m} A_{s} Y_{t-s}+\varepsilon_{t}
$$

Em que $Y_{t}$ é um vetor coluna, de tamanho $7 \times 1$, das volatilidades intradiárias dos sete mercados considerados, $C$ é um vetor coluna, $7 \times 1$, da componente determinística, $A_{s}$ são matrize dos coeficientes estimados, de tamanho $7 \times 7, \mathrm{~m}$ é a ordem de defasagem, e $\varepsilon_{t}$ é o vetor $(7 \times 1)$ dos termos dos resíduos, designado na terminologia do VAR por inovações ou choques.

Previamente à estimação do modelo VAR, foram aplicados os testes para a estacionariedade, uma vez que o modelo empírico, baseado no vetor autorregressivo, pressupõe a estacionariedade das variáveis (Brooks, 2002).

\section{Testes de causalidade}

Com o objetivo de estudar as ligações de curto prazo entre os mercados selecionados e a direção de influência entre esses, recorremos ao conceito de causalidade de Granger, introduzido por Granger (1969), e mais tarde popularizado por Sims (1972), e que assenta no pressuposto de que $X_{t}$ causa $Y_{t}$ se a predição da variável $Y_{t}$ for melhorada com a informação defasada de $X_{t}$, isto é, se a predição de $Y_{t}$ for mais precisa quando usada a informação defasada conjunta de $X_{t}$ e $Y_{t}$, do que considerando apenas a informação de $Y_{t}$. $\mathrm{O}$ teste de causalidade de Granger será obtido a partir do modelo VAR, com o objetivo de saber se as defasagens da variável excluída afetam a variável endógena. Esse teste é designado por VAR Granger Causality / Block Exogenety Wald Tests, 
pressupondo a hipótese nula de que as variáveis endógenas defasadas não causam no sentido de Granger a variável dependente. Importa, contudo, realçar que o resultado desse teste apresenta elevada sensibilidade à ordem de defasagem considerada no modelo (Gujarati, 2003).

\section{Funções de impulso-resposta}

Para estudar as ligações de curto prazo entre os índices dos mercados, são ainda consideradas as funções impulso-resposta. Ao analisarem todas as variáveis introduzidas no sistema, tais funções proporcionam uma análise dinâmica, gerada com base nas estimativas do modelo VAR, permitindo analisar as relações de causalidade verificadas, mesmo quando não sejam detectadas previamente relações de causalidade de Granger entre as variáveis (Lutkepohl,1999).

A função impulso-resposta mostra o modo como determinada variável responde, com o passar do tempo, a um aumento surpresa nessa variável ou noutra variável incluída no modelo VAR, ou seja, uma inovação numa variável desencadeia uma reação em cadeia, ao longo do tempo, nas restantes variáveis do VAR. A função impulso-resposta permite calcular essas reações em cadeia.

Como referem Lutkepohl e Saikkonen (1997) e Aziakpono (2006), se um processo é ruído branco, então o VAR estimado pode ser convertido numa representação de média móvel, cujos coeficientes são impulsos-respostas dos erros de previsão. A média móvel assume a seguinte forma:

$$
Y_{t}=C+\sum_{s=0}^{k} B_{s} \varepsilon_{t-:}
$$

Onde $Y_{t}$ traduz a combinação linear do estado atual e do estado passado dos estímulos dos erros de previsão. No contexto desta abordagem, o coeficiente $\beta_{s}$ pode ser interpretado como a resposta de um índice à inovação de um desvio-padrão, ocorrida há $s$ períodos, em qualquer dos índices sob estudo.
No presente trabalho, optamos por recorrer a funções impulso-resposta generalizadas, introduzidas por Koop; Pesaran e Potter (1996) e Pesaran e Shin (1998), e por escolher o procedimento de simulação de Monte Carlo, com repetição de 1.000 vezes. Esta análise diferencia-se da tradicional análise impulso-resposta ortogonalizada, por não depender da ordenação das variáveis no modelo VAR. A abordagem tradicional, como, por exemplo, a baseada na factorização de Cholesky, para a ortogonalização das inovações do VAR, conduz a diferentes resultados, em função da ordenação de variáveis.

\section{3_Resultados empíricos}

Na Tabela 1, são apresentadas as principais estatísticas descritivas das estimativas de volatilidade intradiária, obtidas por meio da metodologia de Rogers; Satchell e Yoon (1994), para os três subperíodos e os sete mercados europeus. Os valores que constam da tabela permitem a conclusão de que as estimativas de volatilidade intradiária evidenciam sinais de desvio em face da hipótese de normalidade, já que os coeficientes de assimetria e de curtose são estatisticamente diferentes dos de uma distribuição normal, de zero e três, respectivamente. As séries analisadas são leptocúrticas e apresentam caudas assimétricas. Para se confirmar a adequação do ajustamento da distribuição normal às distribuições empíricas das sete séries, foi também aplicado o teste de aderência de Jarque-Bera, ${ }^{1}$ nos três subperíodos considerados na análise. Os valores do teste de aderência, que podem ser vistos na tabela de estatísticas descritivas, permitem a conclusão de que todas as séries são estatisticamente significativas ao nível de significância de 1\%, rejeitando-se claramente a hipótese de normalidade dessas.

No subperíodo Dot-Com, o DAX foi o índice que apresentou volatilidade intradiária média mais elevada, correspondendo ao dobro do valor evidenciado pelos índices FTSE e 
Tabela 1_Estatísticas descritivas da volatilidade intradiária nos três subperíodos

\begin{tabular}{|c|c|c|c|c|c|c|c|c|}
\hline & & ATG & CAC & DAX & FTSE & IBEX & ISEQ & PSI \\
\hline \multirow{9}{*}{$\begin{array}{l}\text { E } \\
\text { ç } \\
\text { ‘े }\end{array}$} & Média & 0,0002 & 0,0002 & 0,0003 & 0,0002 & 0,0002 & 0,0002 & 0,0002 \\
\hline & Mediana & 0,0001 & 0,0001 & 0,0002 & 0,0001 & 0,0001 & 0,0001 & 0,0001 \\
\hline & Máximo & 0,0032 & 0,0035 & 0,0079 & 0,0038 & 0,0023 & 0,0058 & 0,0033 \\
\hline & Mínimo & 0,0000 & 0,0000 & 0,0000 & 0,0000 & 0,0000 & 0,0000 & $-0,0002$ \\
\hline & Desvio-padrão & 0,0003 & 0,0003 & 0,0005 & 0,0003 & 0,0003 & 0,0004 & 0,0003 \\
\hline & Assimetria & 6,3335 & 4,5375 & 6,0809 & 7,1531 & 3,6614 & 9,4394 & 4,8207 \\
\hline & Curtose & 56,9418 & 34,4668 & 66,7215 & 76,9588 & 22,4867 & 116,5564 & 32,9553 \\
\hline & Jarque-Bera & $(0,0000)$ & $(0,0000)$ & $(0,0000)$ & $(0,0000)$ & $(0,0000)$ & $(0,0000)$ & $(0,0000)$ \\
\hline & ADF & $(0,0000)$ & $(0,0000)$ & (0,0000) & $(0,0000)$ & $(0,0000)$ & $(0,0000)$ & $(0,0000)$ \\
\hline \multirow{9}{*}{ 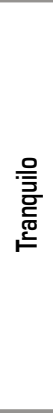 } & Média & 0,0001 & 0,0001 & 0,0001 & 0,0000 & 0,0000 & 0,0001 & 0,0000 \\
\hline & Mediana & 0,0000 & 0,0000 & 0,0000 & 0,0000 & 0,0000 & 0,0000 & 0,0000 \\
\hline & Máximo & 0,0029 & 0,0014 & 0,0011 & 0,0011 & 0,0011 & 0,0052 & 0,0006 \\
\hline & Mínimo & 0,0000 & 0,0000 & 0,0000 & 0,0000 & 0,0000 & 0,0000 & 0,0000 \\
\hline & Desvio-padrão & 0,0001 & 0,0001 & 0,0001 & 0,0001 & 0,0001 & 0,0003 & 0,0000 \\
\hline & Assimetria & 17,2667 & 6,7534 & 3,9287 & 8,3690 & 7,6726 & 10,2722 & 5,0602 \\
\hline & Curtose & 423,4104 & 83,3742 & 24,7367 & 131,1937 & 108,1418 & 129,8529 & 41,5813 \\
\hline & Jarque-Bera & $(0,0000)$ & $(0,0000)$ & $(0,0000)$ & $(0,0000)$ & $(0,0000)$ & $(0,0000)$ & $(0,0000)$ \\
\hline & ADF & (0,0000) & $(0,0000)$ & (0,0000) & $(0,0000)$ & $(0,0000)$ & $(0,0000)$ & $(0,0000)$ \\
\hline \multirow{9}{*}{ 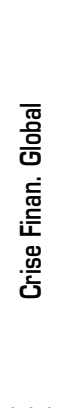 } & Média & 0,0003 & 0,0002 & 0,0002 & 0,0002 & 0,0002 & 0,0003 & 0,0001 \\
\hline & Mediana & 0,0002 & 0,0001 & 0,0001 & 0,0001 & 0,0001 & 0,0001 & 0,0001 \\
\hline & Máximo & 0,0099 & 0,0048 & 0,0056 & 0,0042 & 0,0036 & 0,0343 & 0,0037 \\
\hline & Mínimo & 0,0000 & 0,0000 & 0,0000 & 0,0000 & 0,0000 & 0,0000 & $-0,0018$ \\
\hline & Desvio-padrão & 0,0005 & 0,0004 & 0,0004 & 0,0003 & 0,0003 & 0,0012 & 0,0003 \\
\hline & Assimetria & 9,6127 & 7,1620 & 7,2106 & 6,4157 & 5,3600 & 24,6940 & 7,2279 \\
\hline & Curtose & 147,6867 & 74,6429 & 77,5826 & 62,6202 & 44,2361 & 703,3894 & 86,3371 \\
\hline & Jarque-Bera & (0,0000) & {$[0,0000)$} & (0,0000) & {$[0,0000)$} & (0,0000) & (0,0000) & {$[0,0000)$} \\
\hline & ADF & $(0,0000)$ & $(0,0000)$ & $(0,0000)$ & $(0,0000)$ & $(0,0000)$ & $(0,0000)$ & $(0,0000)$ \\
\hline
\end{tabular}

Fonte: Elaboração própria.

Nota: Os valores entre parênteses dizem respeito aos p-values.

ISEQ, que foram os menos voláteis. Por seu lado, o índice DAX foi o que revelou maior grau de variabilidade na volatilidade intradiária, medida pelo desvio-padrão não condicional.

No subperíodo Tranquilo, caracterizado por uma subida consistente e generalizada dos valores dos índices mun- diais, os índices DAX e ISEQ foram os que evidenciaram níveis mais elevados de volatilidade intradiária. Os restantes mercados apresentaram níveis de volatilidade muito inferiores. Em qualquer dos casos, os valores obtidos ficaram muito abaixo dos verificados no subperíodo Dot-Com. Quanto 
à variabilidade da volatilidade intradiária, o índice ISEQ apresentou os valores mais elevados, enquanto o índice PSI, os mais reduzidos.

Relativamente ao subperíodo Crise Financeira Global, os índices ATG e ISEQ foram os que registraram volatilidade intradiária média mais elevada. Alguns desses valores são, de certo modo, surpreendentes. É o que acontece no caso do índice PSI20, que revelou a volatilidade mais reduzida entre os mercados europeus, embora seja representativo de um pequeno mercado desenvolvido e, sobretudo, objeto de ajuda externa, no ano de 2011, em consequência da crise das dívidas soberanas. Quanto à variabilidade da volatilidade intradiária, essa foi particularmente elevada no índice ISEQ.

Com o objetivo de averiguar a estacionariedade das séries, aplicamos o tradicional teste $\mathrm{ADF}$, de acordo com a equação 4. A hipótese nula do teste estipula que a série tem raiz unitária, ou seja, que a série é integrada de ordem 1,em face da hipótese alternativa de a série não possuir raiz unitária. As séries de volatilidade intradiária evidenciam estacionariedade, nos três subperíodos amostrais. Os valores dos p-values do teste $\mathrm{ADF}$, das diversas séries analisadas são, em todos os casos, inferiores ao nível de significância de $5 \%$, pelo que se rejeita a hipótese nula de integração das séries, concluindo-se que essas são estacionárias.

Para estudar a interação da volatilidade intradiária dos mercados, foram calculadas as matrizes de correlação não condicional, de cada um dos três subperíodos, cujos valores são mostrados nas Tabelas 2,3 e 4. Os valores calculados mostram que as correlações contemporâneas entre os mercados aumentaram ao longo do período estudado. As correlações entre os mercados são positivas, o que sugere a existência de uma tendência/fator comum, que conduz os mercados na mesma direção.

Com base nas matrizes de correlação, foram calculados os rácios de verossimilhança. Em todos os subperíodos, os
Tabela 2_Correlações contemporâneas entre as volatilidades intradiárias dos índices europeus durante o subperíodo Dot-Com

\begin{tabular}{|c|c|c|c|c|c|c|c|}
\hline & ATG & CAC & DAX & FTSE & IBEX & ISEQ & PSI \\
\hline ATG & 1,0000 & & & & & & \\
\hline \multirow[t]{2}{*}{ CAC } & 0,1456 & 1,0000 & & & & & \\
\hline & $(0,0000)$ & & & & & & \\
\hline \multirow[t]{2}{*}{ DAX } & 0,0987 & 0,7570 & 1,0000 & & & & \\
\hline & 0,0060 & $(0,0000)$ & & & & & \\
\hline \multirow[t]{2}{*}{ FTSE } & 0,1386 & 0,7627 & 0,7231 & 1,0000 & & & \\
\hline & 0,0001 & $(0,0000)$ & $(0,0000)$ & & & & \\
\hline \multirow[t]{2}{*}{ IBEX } & 0,2079 & 0,7064 & 0,6868 & 0,6219 & 1,0000 & & \\
\hline & $(0,0000)$ & $(0,0000)$ & $(0,0000)$ & $(0,0000)$ & & & \\
\hline \multirow[t]{2}{*}{ ISEQ } & 0,0313 & 0,1725 & 0,1529 & 0,1537 & 0,1792 & 1,0000 & \\
\hline & 0,3848 & $(0,0000)$ & $(0,0000)$ & $(0,0000)$ & $(0,0000)$ & & \\
\hline \multirow[t]{2}{*}{ PSI } & 0,2488 & 0,3471 & 0,3229 & 0,3348 & 0,3990 & 0,1111 & 1,0000 \\
\hline & {$[0,0000)$} & $(0,0000)$ & $(0,0000)$ & $(0,0000)$ & $(0,0000)$ & $(0,0020)$ & \\
\hline
\end{tabular}

Fonte: Elaboração própria.

Notas: Esta tabela apresenta os coeficientes de correlação entre as volatilidades intradiárias dos índices europeus, no subperíodo Dot-Com, entre 4 de outubro de 1999 e 31 de março de 2003. Os valores entre parênteses dizem respeito aos $p$-values, sendo todos aproximadamente iguais a zero, pelo que todos os coeficientes de correlação são estatisticamente significativos ao nível de $1 \%$.

rácios apresentaram significância estatística, para o nível de significância de $1 \%$, aumentando de valor ao longo de todo o período da amostra. Este fato pode ser interpretado como uma primeira indicação de que, em geral, as correlações entre as volatilidades intradiárias nos mercados estudados tenderam a aumentar. $\mathrm{O}$ aumento do valor do rácio de verossimilhança foi particularmente expressivo no subperíodo Crise Financeira Global (5287), enquanto nos dois subperíodos anteriores os valores foram de 3205 e 3371, evidenciando maior proximidade entre os diversos mercados.

Analisando as correlações não condicionais entre as volatilidades intradiárias dos vários índices europeus, conclui-se que apenas as correlações entre os pares ISEQ-ATG, no 
subperíodo Dot-Com, e ISEQ-ATG e ISEQ-PSI, no subperíodo Tranquilo, não se revelaram estatisticamente significativos. Em todos os restantes casos, as correlações revelaram significância estatística, ao nível de significância de $1 \%$.

No subperíodo Crise Financeira Global, todas as correlações não condicionais foram positivas e estatisticamente significativas, ao nível de significância de 1\%, destacando-se os valores excepcionalmente elevados das correlações observadas entre os pares DAX-FTSE $(0,84)$ e DAX-CAC $(0,83)$. As correlações mais fracas ocorreram nos pares que envolveram o índice ISEQ.

Comparativamente com os dois primeiros subperíodos, os valores das correlações não condicionais, no subperío-

Tabela 3_Correlações contemporâneas entre as volatilidades intradiárias dos índices europeus durante o subperíodo Tranquilo

\begin{tabular}{|c|c|c|c|c|c|c|c|}
\hline & ATG & CAC & DAX & FTSE & IBEX & ISEQ & PSI \\
\hline ATG & 1,0000 & & & & & & \\
\hline \multirow[t]{2}{*}{ CAC } & 0,2293 & 1,0000 & & & & & \\
\hline & $(0,0000)$ & & & & & & \\
\hline \multirow[t]{2}{*}{ DAX } & 0,1508 & 0,7525 & 1,0000 & & & & \\
\hline & $(0,0000)$ & $(0,0000)$ & & & & & \\
\hline \multirow[t]{2}{*}{ FTSE } & 0,2242 & 0,7248 & 0,5770 & 1,0000 & & & \\
\hline & $(0,0000)$ & $(0,0000)$ & $(0,0000)$ & & & & \\
\hline \multirow[t]{2}{*}{ IBEX } & 0,2033 & 0,8176 & 0,6056 & 0,6728 & 1,0000 & & \\
\hline & $(0,0000)$ & $(0,0000)$ & $(0,0000)$ & $(0,0000)$ & & & \\
\hline \multirow[t]{2}{*}{ ISEQ } & 0,0223 & 0,1061 & 0,0943 & 0,2191 & 0,1135 & 1,0000 & \\
\hline & $(0,4657)$ & $(0,0005)$ & 0,0019 & $(0,0000)$ & $(0,0002)$ & & \\
\hline \multirow[t]{2}{*}{ PSI } & 0,1682 & 0,4723 & 0,4280 & 0,3859 & 0,4022 & 0,0374 & 1,0000 \\
\hline & $(0,0000)$ & $(0,0000)$ & $(0,0000)$ & $(0,0000)$ & $(0,0000)$ & (0,2203) & \\
\hline
\end{tabular}

Fonte: Elaboração própria.

Notas: Esta tabela apresenta os coeficientes de correlação entre as volatilidades intradiárias dos índices europeus, no subperíodo Tranquilo, entre $1^{\circ}$ de abril de 2003 e 31 de julho de 2007. Os valores entre parênteses dizem respeito aos p-values, sendo que apenas os pares ISEQ-ATG e ISEQ-PSI não revelaram significância estatística. Todos os restantes pares de correlações mostraram significância estatística, ao nível de $1 \%$. do Crise Financeira Global, sofreram um incremento generalizado. Apenas em dois casos tal não se verificou, concretamente nos pares IBEX-ISEQ e CAC-IBEX, nos subperíodos Dot-Com e Tranquilo, respectivamente.

No sentido de perceber se o aumento generalizado das correlações teve significância estatística, foi aplicado o teste em duas amostras, de acordo com a proposta de Forbes e Rigobon (2002), e que se baseia na transformação de Fisher. Os resultados do teste são apresentados na Tabela 5 , em que é comparado o subperíodo Crise Financeira Global com os dois precedentes.

No subperíodo Crise Financeira Global, em face do subperíodo Tranquilo, os níveis de dependência linear entre os

Tabela 4_Correlações contemporâneas entre as volatilidades intradiárias dos índices europeus durante o período da crise financeira global

\begin{tabular}{|c|c|c|c|c|c|c|c|}
\hline & ATG & CAC & DAX & FTSE & IBEX & ISEQ & PSI \\
\hline ATG & 1,0000 & & & & & & \\
\hline \multirow[t]{2}{*}{ CAC } & 0,6304 & 1,0000 & & & & & \\
\hline & $(0,0000)$ & & & & & & \\
\hline \multirow[t]{2}{*}{ DAX } & 0,6901 & 0,8282 & 1,0000 & & & & \\
\hline & $(0,0000)$ & $(0,0000)$ & & & & & \\
\hline \multirow[t]{2}{*}{ FTSE } & 0,6413 & 0,7630 & 0,8409 & 1,0000 & & & \\
\hline & $(0,0000)$ & $(0,0000)$ & $(0,0000)$ & & & & \\
\hline \multirow[t]{2}{*}{ IBEX } & 0,5095 & 0,7401 & 0,7171 & 0,6903 & 1,0000 & & \\
\hline & $(0,0000)$ & $(0,0000)$ & $(0,0000)$ & $(0,0000)$ & & & \\
\hline \multirow[t]{2}{*}{ ISEQ } & 0,1796 & 0,3596 & 0,2514 & 0,3310 & 0,1700 & 1,0000 & \\
\hline & $(0,0000)$ & $(0,0000)$ & $(0,0000)$ & $(0,0000)$ & (0,0000) & & \\
\hline \multirow[t]{2}{*}{ PSI } & 0,5256 & 0,5677 & 0,6264 & 0,6005 & 0,6345 & 0,2351 & 1,0000 \\
\hline & $(0,0000)$ & $(0,0000)$ & $(0,0000)$ & $(0,0000)$ & $(0,0000)$ & $(0,0000)$ & \\
\hline
\end{tabular}

Fonte: Elaboração própria.

Notas: Essa tabela apresenta os coeficientes de correlação entre as volatilidades intradiárias dos índices europeus, no subperíodo Crise Financeira Global, entre $1^{\circ}$ de agosto de 2007 e 30 de junho de 2011. Os valores entre parênteses dizem respeito aos $p$-values, sendo todos aproximadamente iguais a zero, pelo que todos os coeficientes de correlação são estatisticamente significativos ao nível de significância de $1 \%$. 
diversos mercados alteraram-se de forma substancial. Dos 21 pares de correlações, 20 viram o seu valor aumentar, ante 0 subperíodo precedente. Apenas o par CAC-IBEX diminuiu de valor, sendo essa diminuição estatisticamente significativa (ns 5\%). Dos aumentos verificados, 17 são estatisticamente significativos, ao nível de significância de $5 \%$, enquanto um é significativo ao nível de $10 \%$. Os índices ATG e PSI revelaram aumento de correlações com todos os restantes índices, estatisticamente significativo ao nível de significância de 1\%. Em relação ao subperíodo Dot-Com, os coeficientes de correlação relativos ao subperíodo Crise Financeira Global observaram um aumento generalizado. Dos 21 pares de correlações, 20 aumentaram de valor, e 17 apresentarem aumento estatisticamente significativo, ao nível de significância de 5\%. Tal como aconteceu na comparação dos dois últimos subperíodos, os índices ATG e PSI apresentaram aumentos de correlação com todos os seus pares, estatisticamente significativos ao nível de significância de $1 \%$.

Para analisar a transmissão de volatilidade entre os mercados, foi estimado um modelo VAR, com base nas séries de volatilidade intradiária. A defasagem ótima foi estimada com base nos critérios de informação de AIC e SIC. Contudo, como para as defasagens determinadas pelos critérios de informação, os resíduos dos modelos VAR estimados apresentaram evidências de autocorrelação, foi aumentada a ordem de defasagem, até os resíduos do modelo se mostrarem não autocorrelacionados. Assim, para estimar os modelos VAR, foram escolhidas as ordens de 35, 32 e 38, para cada um dos três subperíodos, respectivamente.

Para contrastar a significância das relações de causalidade, foi testada a hipótese nula de que não existe relação causal entre os índices, com base no procedimento VAR Granger Causality / Block Exogeneity Wald Tests. Esse procedimento avalia a significância conjunta de cada variável endógena defasada, em cada uma das equações do VAR, através
Tabela 5_Teste à igualdade das correlações das volatilidades intradiárias

\begin{tabular}{l|l|l|l|l|l|l}
\multicolumn{6}{c}{ Crise Financeira Global versus Crise Dot-Com } \\
& CAC & DAX & FTSE & IBEX & ISEQ & PSI \\
\hline ATG & 12,3395 & 15,5200 & 12,8649 & 7,2747 & 3,1130 & 6,8345 \\
\hline CAC & & 4,0043 & 0,0163 & 1,4655 & 4,1891 & 5,8420 \\
\hline DAX & & & 6,4250 & 1,2367 & 2,1302 & 8,3020 \\
\hline FTSE & & & & 2,4956 & 3,9164 & 7,1644 \\
\hline IBEX & & & & & $-0,1971$ & 6,7617 \\
\hline ISEQ & & & & $\ldots$ & $\ldots$ & 2,6534 \\
\hline
\end{tabular}

\begin{tabular}{l|l|l|l|l|l|l}
\hline \multicolumn{6}{c}{ Crise Financeira Global versus subperíodo Tranquilo } \\
& CAC & DAX & FTSE & IBEX & ISEQ & PSI \\
\hline ATG & 11,4805 & 15,7115 & 12,0142 & 8,0340 & 3,5948 & 9,3484 \\
\hline CAC & & 4,5986 & 1,9351 & $-4,4871$ & 6,0927 & 2,9585 \\
\hline DAX & & & 12,7824 & 4,5054 & 3,6628 & 6,2759 \\
\hline FTSE & & & & 0,7387 & 2,7357 & 6,4761 \\
\hline IBEX & & & & & 1,3009 & 7,2802 \\
\hline ISEQ & & & & $\ldots$ & $\ldots$ & 4,5630 \\
\hline
\end{tabular}

Fonte: Elaboração própria.

Notas: Essa tabela apresenta os testes à igualdade das correlações entre as volatilidades intradiárias dos índices europeus, tendo por base a transformação de Fisher. Na primeira parte da tabela, é apresentada a comparação entre os subperíodos Crise Financeira Global e Dot-Com, ao passo que, na segunda parte, é apresentada a comparação dos subperíodos Crise Financeira Global e Tranquilo. Valores do teste superiores ou iguais a 1,96 (1,64) são significativos ao nível de significância de 5\% (10\%).

dos valores da estatística $\chi^{2}$, mas também a significância conjunta de todas as variáveis endógenas defasadas na equação, por meio da estatística F. Para cada equação do modelo VAR estimado, são apresentadas as estatísticas do teste Wald, relativas à significância conjunta de cada uma das outras variáveis endógenas, considerando as defasagens selecionadas anteriormente. Os resultados dos testes constam das Tabelas 6, 7 e 8 . 
No primeiro subperíodo, os índices CAC e FTSE foram os mais endógenos, por terem sido causados, no sentido de Granger, por quatro dos seus pares. Por seu lado, o índice mais exógeno foi o IBEX. Já, no segundo subperíodo, os índices ATG e DAX tiveram maior expressão em termos de endogeneidade, enquanto o ISEQ a teve em termos de precedência. Durante o subperíodo Crise Financeira Global, o índice mais exógeno foi o ISEQ, por causar no sentido de Granger todos os restantes índices, enquanto o menos exógeno foi o ATG, tendo apenas causado o índice PSI. Por sua vez, os índices CAC e DAX foram os mais endógenos, ao serem causados por cinco dos seus pares.

Importa também sublinhar que, durante o último subperíodo, cada um dos índices individualmente foi causado, no sentido de Granger, pelos restantes índices de forma conjunta, contrariamente ao que se verificou nos dois subperíodos precedentes. No subperíodo Dot-Com, o índice ATG não foi causado, no sentido de Granger, pelo efeito conjunto dos

Tabela 6_Testes de Causalidade de Granger / Block Exogeneity Wald Tests no subperíodo Dot-Com

\begin{tabular}{|c|c|c|c|c|c|c|c|c|}
\hline & & \multicolumn{7}{|c|}{ Variáveis Dependentes } \\
\hline & & ATG & CAC & DAX & FTSE & IBEX & ISEQ & PSI \\
\hline \multirow{16}{*}{ 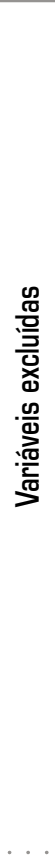 } & \multirow{2}{*}{ ATG } & & 64,9495 & 44,4541 & 61,5752 & 55,3074 & 35,5829 & 100,8904 \\
\hline & & & $(0,0015)$ & $(0,1314)$ & $(0,0036)$ & $(0,0158)$ & $(0,4408)$ & $(0,0000)$ \\
\hline & \multirow{2}{*}{ CAC } & 37,9913 & & 105,0539 & 75,7581 & 65,7610 & 73,4674 & 44,0814 \\
\hline & & $(0,3347)$ & & $(0,0000)$ & $(0,0001)$ & $(0,0013)$ & $(0,0002)$ & $(0,1397)$ \\
\hline & \multirow{2}{*}{ DAX } & 28,5360 & 61,8455 & & 74,5172 & 40,8104 & 37,6118 & 42,8439 \\
\hline & & $(0,7719)$ & $(0,0034)$ & & $(0,0001)$ & (0,2302) & (0,3505) & $(0,1701)$ \\
\hline & \multirow{2}{*}{ FTSE } & 31,2908 & 85,3665 & 87,8796 & & 42,9145 & 164,2331 & 94,1975 \\
\hline & & $(0,6479)$ & $(0,0000)$ & $(0,0000)$ & & (0,1682) & $(0,0000)$ & $(0,0000)$ \\
\hline & \multirow{2}{*}{ IBEX } & 51,3549 & 74,0629 & 55,8255 & 61,8222 & & 41,0614 & 64,3258 \\
\hline & & $(0,0367)$ & $(0,0001)$ & $(0,0141)$ & $(0,0034)$ & & (0,2221) & $(0,0018)$ \\
\hline & \multirow{2}{*}{ ISEQ } & 15,7574 & 37,3465 & 41,4907 & 24,7201 & 31,4004 & & 30,9739 \\
\hline & & (0,9979) & $(0,3618)$ & $(0,2087)$ & $(0,9020)$ & $(0,6426)$ & & $(0,6629)$ \\
\hline & \multirow{2}{*}{ PSI } & 54,1992 & 33,4193 & 48,4380 & 45,2477 & 31,4387 & 88,0708 & \\
\hline & & (0,0202) & $(0,5445)$ & $(0,0649)$ & $(0,1150)$ & $(0,6408)$ & $(0,0000)$ & \\
\hline & \multirow{2}{*}{ Todos } & 201,4030 & 395,7998 & 463,7451 & 367,8992 & 326,4938 & 484,3961 & 478,5143 \\
\hline & & (0,6527) & $(0,0000)$ & $(0,0000)$ & $(0,0000)$ & $(0,0000)$ & $(0,0000)$ & $(0,0000)$ \\
\hline
\end{tabular}

Fonte: Elaboração própria.

Nota: Os valores entre parênteses indicam o $p$-value. 
restantes índices defasados. O mesmo ocorreu no subperíodo Tranquilo, relativamente aos índices IBEX, ISEQ e PSI.

As análises de causalidade permitiram detectar diversas ligações de causalidade unidirecional. Em concreto, foram detectadas 21,10 e 24 relações de causalidade unidirecional com significância estatística, ao nível de significância de 5\%, nos subperíodos Dot-Com, Tranquilo e Crise Financeira Global, respectivamente, o que corresponde a $50 \%$, $24 \%$ e $57 \%$ de ligações entre os pares de mercado estatisticamente significativas, nos três subperíodos. Por outro lado, no segundo subperíodo, foi encontrada apenas uma relação de causalidade mútua, com significância estatística, ao nível de significância de 5\%, entre o par de índices DAX-PSI. No primeiro subperíodo, foram registradas 6 relações de causalidade bidirecional significativas (ns $5 \%$ ), envolvendo os pares ATG-IBEX, ATG-PSI, CAC-DAX, CAC-FTSE, CAC-IBEX e DAX-FTSE. No último subperíodo, o número de relações de causalidade bidirecional aumentou para oito, envolvendo os pares CAC-DAX, CAC-IBEX, CAC-ISEQ, CAC-PSI, DAX-FTSE, DAX-IBEX, FTSE-ISEQ e IBEX-PSI.

Tabela 7_Testes de Causalidade de Granger / Block Exogeneity Wald Tests no subperíodo Tranquilo

\begin{tabular}{|c|c|c|c|c|c|c|c|c|}
\hline & \multicolumn{7}{|c|}{ Variáveis Dependentes } \\
\hline & & ATG & CAC & DAX & FTSE & IBEX & ISEQ & PSI \\
\hline \multirow{16}{*}{ 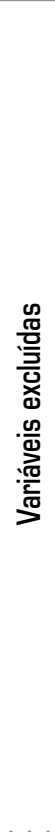 } & \multirow{2}{*}{ ATG } & & 45,7790 & 56,2086 & 36,5111 & 31,9616 & 37,2348 & 38,1312 \\
\hline & & & $(0,0544)$ & $(0,0052)$ & (0,2670) & $(0,4686)$ & $(0,2407)$ & $(0,2106)$ \\
\hline & \multirow{2}{*}{ CAC } & 39,8298 & & 44,4414 & 20,2914 & 23,1107 & 22,4725 & 31,5125 \\
\hline & & $(0,1610)$ & & $(0,0707)$ & $(0,9460)$ & $(0,8748)$ & $(0,8943)$ & $(0,4911)$ \\
\hline & \multirow{2}{*}{ DAX } & 25,4687 & 37,3169 & & 24,4531 & 23,2753 & 8,4849 & 52,5215 \\
\hline & & $(0,7866)$ & $(0,2378)$ & & $(0,8276)$ & $(0,8694)$ & $(1,0000)$ & $(0,0126)$ \\
\hline & \multirow{2}{*}{ FTSE } & 94,2529 & 24,0909 & 34,8795 & & 19,2426 & 28,0785 & 38,1838 \\
\hline & & $(0,0000)$ & $(0,8411)$ & $(0,3327)$ & & (0,9632) & $(0,6655)$ & (0,2089) \\
\hline & \multirow{2}{*}{ IBEX } & 48,8617 & 24,5864 & 49,1906 & २2,2725 & & 41,2869 & 31,0878 \\
\hline & & (0,0286) & (0,8224) & $(0,0266)$ & (0,8999) & & (0,1259) & $(0,5126)$ \\
\hline & \multirow{2}{*}{ ISEQ } & २20,8268 & 91,8680 & 35,2922 & 103,4467 & 79,1265 & & 18,3744 \\
\hline & & $(0,0000)$ & $(0,0000)$ & $(0,3153)$ & $(0,0000)$ & $(0,0000)$ & & $(0,9741)$ \\
\hline & \multirow{2}{*}{ PSI } & 38,9800 & 42,5412 & 63,4679 & 33,2233 & 33,2029 & 13,4773 & \\
\hline & & $(0,1846)$ & $(0,1008)$ & $(0,0008)$ & $(0,4074)$ & $(0,4084)$ & $(0,9983)$ & \\
\hline & \multirow{2}{*}{ Todos } & 494,4077 & 246,1941 & 301,0223 & 250,8365 & 206,8054 & 135,0331 & 193,3393 \\
\hline & & $(0,0000)$ & $(0,0050)$ & $(0,0000)$ & (0,0028) & $(0,2204)$ & $(0,9994)$ & $(0,4593)$ \\
\hline
\end{tabular}

Fonte: Elaboração própria.

Nota: Os valores entre parênteses indicam o $p$-value. 
Os resultados obtidos sugerem que, nos subperíodos de crise, ocorreu um aumento das interdependências de curto prazo entre as volatilidades intradiárias dos índices, com especial ênfase para o subperíodo correspondente à Crise Financeira Global. As ligações entre os mercados foram claramente mais fortes do que as resultantes dos trabalhos de Hamao et al. (1990), Booth et al. (1997) e Caporale et al. (2006), tanto em termos unidirecionais como bidirecionais. Por outro lado, os resultados mostram também que os pressupostos da hipótese do mercado eficiente são questionáveis, uma vez que os movi- mentos ocorridos num determinado mercado são, em parte, precedidos por movimentos passados nos restantes mercados, o que evidencia certa previsibilidade nesses movimentos.

O teste de causalidade de Granger ajuda a perceber as ligações entre os mercados, mas não permite saber se os índices produzem um efeito negativo ou um efeito positivo nos seus pares, nem possibilita saber se umas ligações são mais fortes do que outras. Com esse objetivo, recorremos a funções impulso-resposta generalizadas das volatilidades intradiárias dos índices, de amplitude correspondente a

Tabela 8_Testes de Causalidade de Granger / Block Exogeneity Wald Tests no subperíodo Crise Financeira Global

\begin{tabular}{|c|c|c|c|c|c|c|c|c|}
\hline & \multicolumn{7}{|c|}{ Variáveis Dependentes } \\
\hline & & ATG & CAC & DAX & FTSE & IBEX & ISEQ & PSI \\
\hline \multirow{16}{*}{ 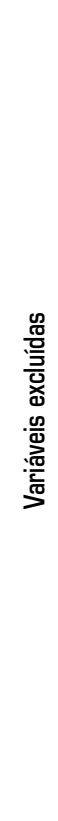 } & \multirow{2}{*}{ ATG } & & 41,0860 & 29,5416 & 27,7927 & 30,4403 & 39,4372 & 69,2358 \\
\hline & & & $(0,3369)$ & $(0,8353)$ & $(0,8883)$ & $(0,8036)$ & {$[0,4055)$} & {$[0,0015)$} \\
\hline & \multirow{2}{*}{ CAC } & 64,8752 & & 60,8177 & 36,3507 & 81,4490 & 90,7324 & 63,2175 \\
\hline & & $(0,0042)$ & & $(0,0108)$ & $(0,5458)$ & $(0,0001)$ & $(0,0000)$ & $(0,0063)$ \\
\hline & \multirow{2}{*}{ DAX } & 40,4592 & 81,2411 & & 63,4475 & 69,5436 & 44,5513 & 36,2849 \\
\hline & & $(0,3623)$ & $(0,0001)$ & & $(0,0059)$ & $(0,0013)$ & $(0,2154)$ & $(0,5489)$ \\
\hline & \multirow{2}{*}{ FTSE } & 25,2675 & 66,9805 & 88,8743 & & 48,9431 & 79,5163 & 24,0299 \\
\hline & & $(0,9437)$ & $(0,0026)$ & $(0,0000)$ & & $(0,1100)$ & $(0,0001)$ & $(0,9622)$ \\
\hline & \multirow{2}{*}{ IBEX } & 43,0964 & 107,3361 & 55,2467 & 45,7949 & & 44,9523 & 64,8228 \\
\hline & & $(0,2624)$ & $(0,0000)$ & 10,03499 & $(0,1802)$ & & $(0,2036)$ & $(0,0043)$ \\
\hline & \multirow{2}{*}{ ISEQ } & 240,8257 & 621,5119 & 507,6580 & 289,6713 & 104,2163 & & 177,3515 \\
\hline & & $(0,0000)$ & $(0,0000)$ & $(0,0000)$ & $(0,0000)$ & $(0,0000)$ & & $(0,0000)$ \\
\hline & \multirow{2}{*}{ PSI } & 29,5977 & 199,7220 & 61,8087 & 42,7218 & 184,3425 & 51,7439 & \\
\hline & & $(0,8334)$ & $(0,0000)$ & $(0,0086)$ & $(0,2754)$ & $(0,0000)$ & $(0,0677)$ & \\
\hline & \multirow{2}{*}{ Todos } & 1515,3360 & 2736,5980 & 2679,1780 & 1171,8990 & 918,4041 & 309,6047 & 796,4247 \\
\hline & & $(0,0000)$ & $(0,0000)$ & $(0,0000)$ & $(0,0000)$ & $(0,0000)$ & $(0,0003)$ & $(0,0000)$ \\
\hline
\end{tabular}

Fonte: Elaboração própria.

Nota: Os valores entre parênteses indicam o $p$-value. 
um desvio-padrão, de modo a obter evidências adicionais acerca dos mecanismos de transmissão dos movimentos de curto prazo dos mercados.

Os resultados das funções impulso-resposta aplicadas às séries de volatilidade intradiária dos índices, nos três subperíodos, são resumidos nas Tabelas 9, 10 e 11.

As funções de resposta a impulsos relativas ao primeiro subperíodo, calculadas com base no modelo de vetores autorregressivos, permitiram identificar 41 casos de reações estatisticamente significativas a choques provenientes do exterior, em 42 casos possíveis (Tabela 9). Apenas a resposta do índice ATG, a impulsos provenientes do mercado irlandês, não teve significância estatística. A maior parte dos choques observados produziu efeitos estatisticamente significativos por mais de um período. Sete choques tiveram efeitos com significância estatística durante um período apenas, em particular os resultantes de impulsos provocados pelo índice irlandês. A esmagadora maioria das reações está de acordo com o sentido esperado, isto é, acompanha o sentido da variação da volatilidade do mercado onde teve origem o choque.

Tabela 9_Resumo dos Resultados das Funções de Resposta a Impulsos obtidas para o subperíodo Dot-Com

\begin{tabular}{|c|c|c|c|c|c|c|c|}
\hline & ATG & CAC & DAX & FTSE & IBEX & ISEQ & PSI \\
\hline \multirow{3}{*}{ ATG } & & 1 & 1 & 2 & 2 & & 2 \\
\hline & & 1 & 1 & 1,2 & 1,2 & & 1,2 \\
\hline & & + & + &,++ &,++ & &,++ \\
\hline \multirow{3}{*}{ CAC } & 3 & & 7 & 7 & 3 & 1 & 4 \\
\hline & $1,5,6$ & & $1,2,3,5,6,7,10$ & $1,2,3,4,6,7,10$ & $1,5,7$ & 1 & $1,3,5,6$ \\
\hline &,,+++ & &,,,,,,+++++++ &,,,,,,+++++++ &,,+++ & + &,,,++++ \\
\hline \multirow{3}{*}{ DAX } & 4 & 7 & & 6 & 4 & 2 & 4 \\
\hline & $1,4,5,6$ & $1,2,3,5,7,8,9$ & & $1,3,5,7,8,9$ & $1,3,5,7$ & 1,10 & $1,3,4,5$ \\
\hline &,,,++++ &,,,,,,+++++++ & &,,,,,++++++ &,,,++++ &,+- &,,,++++ \\
\hline \multirow{3}{*}{ FTSE } & 4 & 5 & 3 & & 3 & 1 & 3 \\
\hline & $1,5,6,7$ & $1,5,6,7,9$ & $1,3,5$ & & $1,5,7$ & 1 & $1,3,5$ \\
\hline &,,,++++ &,,,,+++++ &,,+++ & &,,+++ & + &,,+++ \\
\hline \multirow{3}{*}{ IBEX } & 4 & 7 & 5 & 8 & & 1 & 4 \\
\hline & $1,2,5,6$ & $1,3,4,5,7,9,10$ & $1,3,4,5,7$ & $1,2,3,4,5,7,9,10$ & & 1 & $1,3,5,7$ \\
\hline &,,,++++ &,,,,,,+++++++ &,,,,+++++ &,,,,,,,++++++++ & & + &,,,++++ \\
\hline \multirow{3}{*}{ ISEQ } & 1 & 2 & 1 & 2 & 2 & & 3 \\
\hline & 2 & 1,3 & 1 & 1,6 & 1,7 & & $1,5,8$ \\
\hline & + &,++ & + &,++ &,++ & &,,+++ \\
\hline \multirow{3}{*}{ PSI } & 4 & 6 & 4 & 6 & 4 & 2 & \\
\hline & $1,2,5,7$ & $1,3,4,5,7,9$ & $1,3,5,9$ & $1,3,4,5,7,9$ & $1,3,5,9$ & 1,2 & \\
\hline & $\ldots++,+,+,+$ & .,,,,,++++++ &,,,,++++ &.,,,,,+++++++ &.,,,++++ &,++ & \\
\hline
\end{tabular}

Fonte: Elaboração própria.

Notas: Respostas dos mercados em linha a impulsos provenientes dos mercados em coluna; cada célula tem três linhas: na primeira linha, indica-se o número de períodos durante os quais a resposta do mercado em linha teve significância estatística de acordo com o critério de um desvio-padrão; na segunda linha, indica-se o número de ordem desses períodos; na terceira linha, indica-se o sinal da resposta ao impulso; a célula não preenchida corresponde à ausência de resposta com significância estatística, de acordo com o critério definido de um desvio-padrão. 
Na Tabela 10, é apresentado o resumo das funções de resposta a impulsos para o subperíodo Tranquilo, calculadas valendo-se do modelo VAR.

Durante o subperíodo Tranquilo, as relações entre os mercados estudados revelaram-se, em geral, significativas (41 em 42 possíveis). São de realçar, no entanto, alguns casos mais notáveis. $\mathrm{O}$ mercado irlandês não foi influenciado significativamente pelo mercado grego. É ainda de salientar o fato de, no mercado Irlandês, os choques mostrarem significância estatística por apenas um período. Também no caso do mercado português, os choques se revelaram, em geral, menos persistentes do que nos restantes índices, com exceção do mercado irlandês, desvanecendo-se por completo ao fim de duas a três sessões de negociação.

Tal como aconteceu nos subperíodos precedentes, a larga maioria das reações está de acordo com o sentido esperado, acompanhando o sinal da variação da volatilidade do mercado onde teve origem o choque.

Tabela 10_Resumo dos Resultados das Funções de Resposta a Impulsos obtidas para o subperíodo Tranquilo

\begin{tabular}{|c|c|c|c|c|c|c|c|}
\hline & ATG & CAC & DAX & FTSE & IBEX & ISEQ & PSI \\
\hline \multirow{3}{*}{ ATG } & & 3 & 4 & 6 & 3 & 3 & 2 \\
\hline & & $1,2,4$ & $1,2,4,7$ & $1,2,4,5,6,7$ & $1,2,4$ & $4,6,7$ & 1,3 \\
\hline & &,,+++ &,,,++++ &,,,,,++++++ &,,+++ &,,+++ &,++ \\
\hline \multirow{3}{*}{ CAC } & 4 & & 6 & 3 & 2 & 3 & 5 \\
\hline & $1,2,3,5$ & & $1,2,3,5,6,7$ & $1,2,7$ & 1,2 & $1,4,9$ & $1,2,7,8,9$ \\
\hline &,,,+++- & &,,,,,++++++ &,,+++ &,++ &,,+++ &,,,,+++++ \\
\hline \multirow{3}{*}{ DAX } & 3 & 4 & & 4 & 4 & 1 & 6 \\
\hline & $1,2,5$ & $1,2,3,7$ & & $1,2,3,7$ & $1,2,3,7$ & 1 & $1,2,3,7,8,9$ \\
\hline &,,++- &,,,++++ & &,,,++++ &,,,++++ & + &,,,,,++++++ \\
\hline \multirow{3}{*}{ FTSE } & 3 & 2 & 2 & & 2 & 2 & 4 \\
\hline & $1,2,7$ & 1,2 & 1,2 & & 1,2 & 1,9 & $1,2,7,9$ \\
\hline &,,+++ &,++ &,++ & &,++ &,++ &,,,++++ \\
\hline \multirow{3}{*}{ IBEX } & 4 & 2 & 5 & 2 & & 3 & 6 \\
\hline & $1,2,3,5$ & 1,2 & $1,2,3,6,7$ & 1,2 & & $1,4,9$ & $1,2,3,7,8,9$ \\
\hline &,,,+++- &,++ &,,,,+++++ &,++ & &,,+++ &,,,,,++++++ \\
\hline \multirow{3}{*}{ ISEQ } & & 1 & 1 & 1 & 1 & & 1 \\
\hline & & 1 & 1 & 1 & 1 & & 1 \\
\hline & & + & + & + & + & & + \\
\hline \multirow{3}{*}{ PSI } & 2 & 2 & 3 & 2 & 2 & 1 & \\
\hline & 1,2 & 1,2 & $1,2,3$ & 1,7 & 1,2 & 1 & \\
\hline &,++ &,++ &,,+++ & $\stackrel{+,+}{.}$ & $\stackrel{+,+}{+}$ & + & \\
\hline
\end{tabular}

Fonte: Elaboração própria.

Notas: Respostas dos mercados em linha a impulsos provenientes dos mercados em coluna; cada célula tem três linhas: na primeira linha, indica-se o número de períodos durante os quais a resposta do mercado em linha teve significância estatística de acordo com o critério de um desvio-padrão; na segunda linha, indica-se o número de ordem desses períodos; na terceira linha, indica-se o sinal da resposta ao impulso; a célula não preenchida corresponde à ausência de resposta com significância estatística, de acordo com o critério definido de um desvio-padrão. 
O resumo das Funções de Resposta a Impulsos para o subperíodo Crise Financeira Global é apresentado na Tabela 11. À semelhança do que aconteceu nos dois subperíodos precedentes, 41 choques mostraram significância estatística. Apenas a resposta do ISEQ a impulsos do ATG não se mostrou estatisticamente significativa.

O número de choques estatisticamente significativos foi de 145, 120 e 155, nos subperíodos Dot-Com, Tranquilo e Crise
Financeira Global, respectivamente, razão para se concluir que, nas fases de volatilidade mais acentuada, o número de reações a choques sobre a volatilidade intradiária, estatisticamente significativas, aumentou em face do subperíodo amostral menos turbulento, o mesmo acontecendo com a persistência dos choques, a revelar-se claramente superior nos dois referidos subperíodos. Por sua vez, a persistência dos choques, quando comparada com a encontrada nou-

Tabela 11_Resumo dos Resultados das Funções de Resposta a Impulsos obtidas para o subperíodo Crise Financeira Global

\begin{tabular}{|c|c|c|c|c|c|c|c|}
\hline & ATG & CAC & DAX & FTSE & IBEX & ISEQ & PSI \\
\hline \multirow{3}{*}{ ATG } & & 4 & 4 & 4 & 2 & 2 & 3 \\
\hline & & $1,2,3,8$ & $1,2,3,4$ & $1,2,3,4$ & 1,2 & 3,8 & $1,2,3$ \\
\hline & &,,,++++ &,,,++++ &,,,++++ &,++ &,++ &,,+++ \\
\hline \multirow{3}{*}{ CAC } & 6 & & 5 & 5 & 5 & 2 & 6 \\
\hline & $1,2,3,7,8,10$ & & $1,2,3,4,7$ & $1,2,3,4,8$ & $1,2,3,4,5$ & 1,5 & $1,2,3,5,6,7$ \\
\hline &,,,,,++++++ & &,,,,+++++ &,,,,+++++ &,,,,+++++ &,+- &,,,,,++++++ \\
\hline \multirow{3}{*}{ DAX } & 4 & 5 & & 5 & 5 & 2 & 7 \\
\hline & $1,2,3,4$ & $1,2,3,4,8$ & & $1,2,3,4,8$ & $1,2,3,4,8$ & 1,4 & $1,2,3,4,5,6,8$ \\
\hline &,,,++++ &,,,,+++++ & &,,,,+++++ &,,,,+++++ &,+- &,,,,,,+++++++ \\
\hline \multirow{3}{*}{ FTSE } & 4 & 4 & 4 & & 4 & 2 & 5 \\
\hline & $1,2,3,4$ & $1,2,3,8$ & $1,2,3,4$ & & $1,2,3,8$ & 1,8 & $1,2,3,6,8$ \\
\hline &,,,++++ &,,,++++ &,,,++++ & &,,,++++ &,++ &,,,,+++++ \\
\hline \multirow{3}{*}{ IBEX } & 4 & 5 & 3 & 4 & & 2 & 7 \\
\hline & $1,4,8,9$ & $1,2,3,6,10$ & $1,2,3$ & $1,2,3,4$ & & 4,5 & $1,2,3,5,6,8,9$ \\
\hline &,,,++++ &,,,,+++++ &,,+++ &,,,++++ & &,-- &,,,,,,+++++++ \\
\hline \multirow{3}{*}{ ISEQ } & & 4 & 4 & 5 & 1 & & 4 \\
\hline & & $1,2,5,10$ & $1,3,4,5$ & $1,2,3,4,5$ & 5 & & $1,2,3,5$ \\
\hline & &,,,++++ &,,,++++ &,,,,+++++ & + & &,,,++++ \\
\hline \multirow{3}{*}{ PSI } & 5 & 4 & 2 & 2 & 1 & 1 & \\
\hline & $1,2,3,4,6$ & $1,3,5,9$ & 1,3 & 1,3 & 1 & 1 & \\
\hline &,,,,+++++ &,,,++++ &,++ &,++ & + & + & \\
\hline
\end{tabular}

Fonte: Elaboração própria.

Notas: Respostas dos mercados em linha a impulsos provenientes dos mercados em coluna; cada célula tem três linhas: na primeira linha, indica-se o número de períodos durante os quais a resposta do mercado em linha teve significância estatística de acordo com o critério de um desvio-padrão; na segunda linha, indica-se o número de ordem desses períodos; na terceira linha, indica-se o sinal da resposta ao impulso; a célula não preenchida corresponde à ausência de resposta com significância estatística, de acordo com o critério definido de um desvio-padrão. 
tros estudos, como, por exemplo, no de Yang et al. (2003), que evidenciou a existência de choques positivos com significância estatística, embora se extinguindo ao fim de um a dois dias, mostrou-se no presente estudo mais elevada, pelo que as informações relativas aos mercados analisados não foram incorporadas instantaneamente, contrariando a hipótese do mercado eficiente. Essas situações refletem maior interdependência entre os mercados, o que reduz as possibilidades de implementação de uma estratégia de diversificação entre os sete mercados.

\section{4_Resumo e conclusões}

Neste trabalho, foram estudadas as ligações entre índices de bolsas europeias, desde a crise das empresas tecnológicas até à atual crise financeira, vista por diversos autores como a mais severa crise financeira após a Grande Depressão e a primeira crise financeira global que o mundo conheceu. $\mathrm{O}$ período amostral foi subdividido em três, dois correspondentes a fases de crise e outro relativo a uma fase de crescimento dos valores dos índices. Recorrendo a uma estimativa de volatilidade intradiária, foram calculadas correlações contemporâneas, estimados vetores autorregressivos e aplicados testes de causalidade e funções de impulso-resposta, com o propósito de perceber se a crise financeira global implicou alterações ao nível das ligações de curto prazo e dos mecanismos de transmissão de volatilidade intradiária dos mercados europeus.

Os valores das correlações contemporâneas entre os mercados aumentaram ao longo do período analisado e são todos positivos. Comparando os pares de correlações nos três subperíodos analisados, conclui-se que o aumento generalizado dessas revelou significância estatística. Adicionalmente, os rácios de verossimilhança apresentaram significância estatística e registraram forte aumento de valor. A conjugação desses elementos sugere a existência de um fator comum, que conduz os mercados na mesma direção.

Os resultados dos testes de causalidade de Granger sugerem um aumento das ligações de curto prazo entre os mercados europeus, quer unidirecional, quer bidirecionalmente, via volatilidade intradiária, durante os subperíodos de crise, com especial ênfase para o correspondente à crise financeira global. Por sua vez, as funções de impulso-resposta mostraram a existência de choques positivos, com significância estatística, com persistência superior a uma semana. Podemos, portanto, inferir que o pressuposto da hipótese da eficiência dos mercados é questionável, uma vez que a previsão do movimento de determinado mercado pode ser melhorada se considerados os movimentos defasados dos restantes mercados, possibilitando a ocorrência de operações de arbitragem.

\section{Nota}

${ }^{1}$ De acordo com este teste, sob a hipótese da normalidade, $J B=N\left[A^{2} / 6+(C-3)^{2} / 24\right]$, segue assintoticamente uma distribuição QuiQuadrado, com 2 graus de liberdade, onde $A$ e $C$ representam, respectivamente, os coeficientes de assimetria e curtose amostrais, e $N$, a dimensão da amostra. 
ALIZADEH, S.; BRANDT, M.; DIEBOLD, F. Range-based estimator of stochastic volatility models.

Working Paper, University of Pennsylvania, 1999.

ANDERSEN, T.; BOLLERLEV, T. Answering the skeptics: Yes, standard volatility models do provide accurate forecasts. International Economic Review, 39, p. 885-905, 1998.

\section{AZIAKPONO, M. Financial} Integration amongst the SACU Countries: Evidence from Interest Rate Pass-Through Analysis. Studies in Economics and Econometrics, v. 30, n. 2, 2006.

BEKAERT, G.; EHRMANN, M.; FRATZSCHER, M.; MEHL, A. Global Crises and Equity Market Contagion. National Bureau of Eeconomic Rerearch. Working Paper 17121. 2011. Disponível em: <http://www.nbs.rs/ export/sites/default/internet/ latinica/90/90_9/Michael_ Ehrmann_wp.pdf $>$. Acesso em: jan. 2013 .

BELTRATTI, A.; MORANA, C. Computing Value at Risk With High Frequency Data. Journal of Empirical Finance, 6, p. 431-455, 1999.

BOLLERSLEV, T. Generalized Autoregressive Conditional Heteroscedasticity. Journal of Econometrics, 31, p. 309-328, 1986.
BOOTH, G.; MARTIKAINEN, T.; TSE, Y. Price and volatility spillovers in Scandinavian stock markets. Journal of Banking and Finance, 21, p. 811-823, 1997.

BROOKS, C. Introductory

Econometrics for Finance.

Cambridge, United Kingdom: Cambridge University Press, 2002.

CAPORALE, G.; PITTIS, N.; SPAGNOLO, N. Volatility transmission and financial crises. Journal of Economics and Finance, v. 30, n. 3, p. 376-390, 2006

CLAESSENS. S.; DELL'ARICCIA,

G.; IGAN, D.; LAEVEN, L. Lessons and Policy Implications from the Global Financial Crisis, IMF Working

Paper No. 10/44, 2010.

DICKEY, D.; FULLER, W. Distribution of the estimators for autoregressive time series with a unit root. Journal of the American Statistical Association, 74, p. 427-431, 1979.

EUN, C.; SHIM, S. International transmission of stock market movements. Journal of Financial and Quantitative Analysis, 24, p. 241-256, 1989.

FAMA, E. Efficient capital markets: A review of empirical work. Journal of Finance, 25, p. 383-417, 1970.
FORBES, K.; RIGOBON,

R. No Contagion, Only Interdependence:

Measuring Stock Market Comovements. Journal of Finance, 57, p. 2223-62, 2002.

GALLANT, A.; HSU, R.; TAUCHEN, G. Using daily range data to calibrate volatility diffusion and extract the forward integrated variance. Working Paper, University of North Carolina, Chapel Hill, 1999.

GRANGER, C. Investigating causal relationships by econometric models and crossspectral methods. Econometrica, v. 37, n. 3, p. 424-438, 1969.

GUJARATI, D. Basic Econometrics, McGraw-Hill, 2003.

HAMAO, Y.; MASULIS, R.; NG, V. Correlations in price changes and volatility across international stock markets. Review of Financial Studies, v. 3 , n. 2, p. 281-307, 1990.

HORTA, P.; MENDES, C; VIEIRA, I. Contagion Effects of the U.S. Subprime Crisis on Developed Countries. Working Paper, University of Évora, 2008.

KOOP, G.; PESARAN, M.; POTTER, $\mathrm{S}$. Impulse response analysis in nonlinear multivariate models. Journal of Econometrics, 74, p. 119147, 1996.
LIN, J.; TREICHEL, V. The Unexpected Global Financial Crisis Researching Its Root Cause. World Bank. WPS5937, 2012. Disponível em: <http://www-wds. worldbank.org/servlet/WDS ContentServer/WDSP/ IB/2012/o 1/o9/000158349_2012010908594 2/Rendered/PDF/WPS5937.pdf $>$. Acesso em: nov. 2012.

LUTKEPOHL, H. Vetor Autoregressions. Unpublished manuscript, Institut fur Statistik und Okonometrie, HumboldtUniversitat zu Berlin, 1999.

LUTKEPOHL, H.; SAIKKONEN, P. Impulse Response Analysis in In.nite Order Cointegrated Vetor Autoregressive Processes. Journal of Econometrics, 81, p. 127-157, 1997.

NAOUI, K.; LIOUANE, N.; BRAHIM, S. A dynamic correlation analysis of financial contagion: the case of the subprime crisis. International Journal of Economics and Finance, v. 2, n. 3 , p. $85-96,2010$

PESARAN, M.; SHIN, Y. Generalized impulse response analysis in linear multivariate models. Economic Letters, 58, p. 17-29, 1988.

PINDYCK, R.; ROTEMBERG. J. The Excess Co-Movement of Commodity Prices. The Economic Journal, p. 1173-89, 1990. 
POON, S.; GRANGER, C.

Forecasting Volatility in

Financial Markets: A Review.

Journal of Economic Literature, v. 41,

n. 2, 80 pages, 2003.

ROGERS, L.; SATCHELL, S.; YOON, Y. Estimating the volatility of stock prices: a comparison of methods that use high and low prices. Applied Financial Economics, 4, p. 241-47, 1994.

SIMS, C. Money, Income and Causality. American Economic Review, 62, p. 540-552, 1972.

SIMS, C. Macroeconomics and reality. Econometrica, 48, p. 1-48, 1980.

TAYLOR, S. Financial returns modelled by the product of two stochastic processes, a study of daily sugar prices, 1969-79. In: Time Series Analysis: Theory and Practice 1, O.D. Anderson editor, North Holland, Amsterdam, p. 203-226, 1982.

TOUSSAINT, E. The US Subprime Crisis Goes Global. Counterpunch, Weekend Edition, January 1213, 2008.

YANG, J; MIN, I.; LI, Q. European Stock Market Integration: Does EMU Matter? Journal of Business

Finance and Accounting, v. 30, n. 9, p. 1253-1276, 2003.

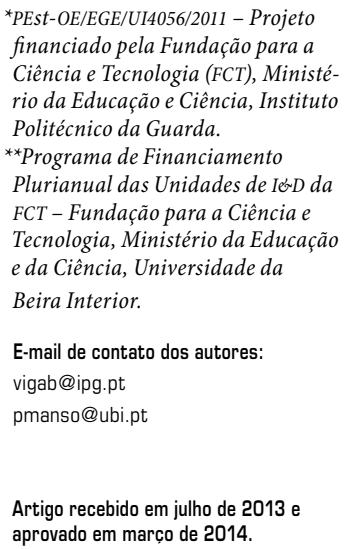


